2016-08-13

\title{
Keratoconus Management: Role of GP Contact Lenses
}

Martin Herranz, R

http://hdl.handle.net/10026.1/5268

$10.4172 / 2155-9570.1000 \mathrm{e} 117$

J Clin Exp Ophthalmol

All content in PEARL is protected by copyright law. Author manuscripts are made available in accordance with publisher policies. Please cite only the published version using the details provided on the item record or document. In the absence of an open licence (e.g. Creative Commons), permissions for further reuse of content should be sought from the publisher or author. 


\section{Journal of Clinical \& Experimental Ophthalmology}

\section{Keratoconus Management: Role of GP Contact Lenses}

\section{Raul Martin Herranz}

Optometry Research Group, IOBA Eye Institute, University of Valladolid, Spain

*Corresponding author: Raul Martin Herranz, Optometry Research Group, IOBA Eye Institute, University of Valladolid, Spain, E-mail: raul@ioba.med.uva.es

Received date: July 07, 2016; Accepted date: July 11, 2016; Published date: July 13, 2016

Copyright: (c) 2016 Herranz RM, et al. This is an open-access article distributed under the terms of the Creative Commons Attribution License, which permits unrestricted use, distribution, and reproduction in any medium, provided the original author and source are credited.

Citation: Herranz RM (2016) Keratoconus Management: Role of GP Contact Lenses. J Clin Exp Ophthalmol 7: e117. doi:10.4172/2155-9570.1000e117

\section{Editorial}

Keratoconus is a corneal disorder progressive characterized by thinning and steepening of the central and paracentral cornea, which leads to protrusion [1]. The exact cause of the disease remains to be fully elucidated although its etiology probably is multifactorial with a genetic predisposition to keratoconus influenced by external environmental factors (eye rubbing and atopy) [2] with different systemic involvement [3]. In the early stages, keratoconus can be managed with spectacles or contact lenses (CL), but when keratoconus progresses, other surgical techniques are often required.

For example, anterior lamellar (dDALK) or penetrating keratoplasty (PK) permit than 3 of 4 patients achieve best-corrected visual acuity of $20 / 40$ or better [4]. Intracorneal ring segment (ICRS) are proposed to increase corneal stability and decrease the astigmatism asymmetry normalizing the corneal contour with slight improvement of patients' visual acuity $[5,6]$. Corneal collagen cross-linking (CXL) is a common technique proposed to reduce disease progression with a light improvement of visual acuity (1 to 2 Snellen lines) [7].

However, visual rehabilitation of keratoconus patients with gaspermeable (GP) CL permits to improve patients' visual acuity until levels near to 20/208; masking corneal irregular astigmatism improving patients' vision. This management's option allows improving patients' quality of live $[8,9]$ and delays the need of corneal graft. Unfortunately, GP CL wear do not stop disease progression [6].

So, future challenges in keratoconus management could require two major milestones; proposing a safe, secure and effective technique to reduce disease progression (minimizing the economic cost to patients and payers, especially if corneal graft will be necessary [10]) and improving GP CL fitting techniques to simplify and facilitate this correction in early or mild disease stages improving the visual rehabilitation. A significant new on-line open-access tool (www.calculens.com) has been recently developed to help eye care practitioners in keratoconus patient management fitting GP CL.

In summary, GP CL remains fundamental in visual rehabilitation of keratoconus patient representing the major nonsurgical option to manage these patients. Therefore, co-management between Ophthalmologist, CL practitioners and Optometrist will help to provide best care to keratoconus patients to avoid or delay the need of corneal transplantation improving patient quality of life.

\section{References}

1. Gomes JA, Tan D, Rapuano CJ, Belin MW, Ambrósio R, et al. (2015) Global consensus on keratoconus and ectatic diseases. Cornea 34: 359-369.

2. McGhee CN, Kim BZ, Wilson PJ (2015) Contemporary treatment paradigms in Keratoconus. Cornea 34: S16- S23.

3. Bahar I, Vinker S, Livny E, Kaiserman I (2010) Possible Association between Keratoconus and Renal Diseases. J Clinic Experiment Ophthalmol 1:112.

4. Arnalich-Montiel F, Alió del Barrio JL, Alió JL (2016) Corneal surgery in keratoconus: which type, which technique, which outcomes? Eye and Vision 3:2.

5. Poulsen DM, Kang JJ (2015) Recent advances in the treatment of corneal ectasia with intrastromal corneal ring segments. Curr Opin Ophthalmol 26: 273-277.

6. Mandathara PS, Stapleton FJ, Willcox MD (2016) Outcome of Keratoconus Management: Review of the Past 20 Years' Contemporary Treatment Modalities. Eye Contact Lens 11.

7. Meiri Z, Keren S, Rosenblatt A, Sarig T, Shenhav L, et al. (2016) Efficacy of corneal collagen cross-linking for the treatment of Keratoconus: A systematic review and Meta-Analysis. Cornea 35: 417-428.

8. Visser ES, Wisse RP, Soeters N, Imhof SM, Van der Lelij A (2016) Objective and subjective evaluation of the performance of medical contact lenses fitted using a contact lens selection algorithm. Cont Lens Anterior Eye 22: S1367-0484.

9. Ortiz-Toquero S, Perez S, De Juan V, Rodriguez G, Agustin Mayo-Iscar A, et al. (2015) The influence of the refractive correction on the Vision-Related Quality of Life in keratoconus patients. Quality of Life Research 251043-251051.

10. Rebenitsch RL, Kymes SM, Walline JJ, Gordon MO (2011) The lifetime economic burden of keratoconus: a decision analysis using a markov model. Am J Ophthalmol 151: 768-773. 